\title{
Six-month outcomes following venovenous ECMO for severe COVID-19 and viral pneumonitis: 2019-2020 Australian experience
}

Aidan JC Burrell, Ary Serpa Neto, Andrew Udy, Vincent Pellegrino and Carol Hodgson

Pandemic viral respiratory tract infections can lead to severe viral pneumonitis which is refractory to conventional intensive care unit (ICU) treatments, necessitating venovenous extracorporeal membrane oxygenation (VVECMO). Over the past 3 years, Australia has experienced two major viral outbreaks, including a non-coronavirus disease 2019 (COVID-19) (predominantly influenza) outbreak in 2019-2020 and the COVID-19 pandemic in 2020. ${ }^{1}$ Few studies have directly compared the patient characteristics, disease course, or long term impact and resource use of these different viral diseases in VV-ECMO patients.

We performed a multicentre, observational study using the Australian national ECMO registry (NCT03793257). We compared patients with severe COVID-19 pneumonitis who were supported by VV-ECMO with patients with nonCOVID-19 (predominantly influenza) viral pneumonitis. Sixmonth survival, as well as disability (World Health Organization Disability Assessment Schedule [WHODAS]) and quality of life (Five-level EuroQol five dimensions questionnaire [EQ-5D-5L]) were assessed by trained, central assessors.

From 30 March 2019 to 31 December 2020, 13 patients were initiated on VV-ECMO for COVID-19 and 23 were initiated for non-COVID-19 viral pneumonitis in seven centres (Table 1). In comparison to non-COVID-19 viral pneumonitis, COVID-19 patients were older (median age, 58 [interquartile range (IQR), 56-66] v 44 [IQR, 33-54] years), but illness severity, arterial partial pressure of oxygen to fraction of inspired oxygen $\left(\mathrm{PaO}_{2}: \mathrm{FiO}_{2}\right)$ ratio, and driving pressure at the time of ECMO initiation were similar (Table 1). Median time from intubation to ECMO initiation was longer in patients with COVID-19 (7.4 [IQR, 3.3-14.0] v 0.7 [IQR, 0.2-4.6] days). Patients with COVID-19 also had increased duration of mechanical ventilation (30.4 v 16.7 days), ICU length of stay (45.6 $v 23.8$ days) and hospital length of stay (55.7 v 30.7 days) compared with viral pneumonitis patients. At 6 months, the mortality was numerically lower in the COVID-19 group (25\% $\vee 38.1 \% ; P=0.70)$ and the incidence of complications were similar. Functional outcomes at 6 months were available in 6/9 and 11/13 of the survivors respectively. Disability and health-related

\begin{abstract}
Objective: To compare the characteristics, treatments and 6-month functional outcomes of patients with coronavirus disease 2019 (COVID-19) versus non-COVID-19 viral pneumonitis supported by venovenous extracorporeal membrane oxygenation (VV-ECMO).

Design: Prospective, observational cohort study in seven intensive care units (ICUs) across Australia.

Participants: Patients admitted to participating ICUs with laboratory-confirmed COVID-19 or viral pneumonitis requiring $\mathrm{VV}$-ECMO.

Results: From 30 March 2019 to 31 December 2020, 13 patients were initiated on VV-ECMO for COVID-19 and 23 were initiated for non-COVID-19 viral pneumonitis. Patients with COVID-19 were older and had a longer duration from intubation to ECMO initiation, but had similar illness severity and APACHE IV scores at the time of initiation. Overall disability, health-related quality of life, and mortality were similar, but ICU and hospital length of stay were significantly longer in patients with COVID-19.

Conclusions: Six-month functional outcomes and mortality were similar between COVID-19 and viral pneumonitis patients treated with VV-ECMO. However, length of stay was longer in COVID-19 patients, which may have resource implications.
\end{abstract}

Crit Care Resusc 2022; 24 (1): 83-6

quality of life were similar. No patients from either group underwent lung transplantation.

This nationwide study highlights important demographic and long term outcome differences in VV-ECMO patients with different types of viral pneumonitis. Although the patients had similar illness severity at baseline, COVID-19 patients were initiated on ECMO much later following 
Table 1. Baseline characteristics of patients with coronavirus disease 2019 (COVID-19) or other viral pneumonitis receiving extracorporeal membrane oxygenation (ECMO)

\begin{tabular}{|c|c|c|c|c|}
\hline & Overall & COVID-19 & Viral pneumonitis & $P$ \\
\hline Total number of patients & 36 & 13 & 23 & \\
\hline Age, years, median (IQR) & $50(37-60)$ & $58(56-66)$ & $44(33-54)$ & 0.005 \\
\hline Sex, female & $10(27.8 \%)$ & $5(38.5 \%)$ & $5(21.7 \%)$ & 0.440 \\
\hline APACHE IV, median (IQR) & $47(33-84)$ & $40(15-64)$ & $48(36-86)$ & 0.277 \\
\hline \multicolumn{5}{|l|}{ SOFA, median (IQR) } \\
\hline Total & $9(6-12)$ & $7(5-10)$ & $9(8-12)$ & 0.393 \\
\hline Respiratory & $4(4-4)$ & $3(3-4)$ & $4(4-4)$ & 0.001 \\
\hline Coagulation & $0(0-1)$ & $0(0-0)$ & $0(0-1)$ & 0.524 \\
\hline Liver & $0(0-0)$ & $0(0-0)$ & $0(0-1)$ & 0.568 \\
\hline Cardiovascular & $3(1-4)$ & $2(1-4)$ & $3(2-4)$ & 0.350 \\
\hline Renal & $1(0-2)$ & $0(0-1)$ & $1(0-2)$ & 0.135 \\
\hline Days between ICU admission and ECMO start, median (IQR) & $5.2(0.9-11.5)$ & $13.6(7.4-16.8)$ & $1.8(0.3-6.1)$ & 0.001 \\
\hline Days between intubation and ECMO start, median (IQR) & $3.3(0.4-7.3)$ & $7.4(3.3-14.0)$ & $0.7(0.2-4.6)$ & 0.006 \\
\hline Type of support & & & & 0.361 \\
\hline VA-ECMO & $1(2.8 \%)$ & $1(7.7 \%)$ & $0(0.0 \%)$ & \\
\hline VV-ECMO & $35(97.2 \%)$ & $12(92.3 \%)$ & $23(100.0 \%)$ & \\
\hline Body mass index, kg/m², median (IQR) & $29.9(25.7-34.3)$ & $27.5(23.9-32.7)$ & $30.4(26.2-35.6)$ & 0.246 \\
\hline Charlson Comorbidity Score, median (IQR) & $1(0-2)$ & $2(2-3)$ & $0(0-1)$ & 0.002 \\
\hline \multicolumn{5}{|l|}{ Comorbidities } \\
\hline Heart failure & $0(0.0 \%)$ & $0(0.0 \%)$ & $0(0.0 \%)$ & - \\
\hline Chronic obstructive pulmonary disease & $1(2.8 \%)$ & $0(0.0 \%)$ & $1(4.3 \%)$ & 0.999 \\
\hline Diabetes & $5(13.9 \%)$ & $4(30.8 \%)$ & $1(4.3 \%)$ & 0.047 \\
\hline Chronic kidney disease & $0(0.0 \%)$ & $0(0.0 \%)$ & $0(0.0 \%)$ & - \\
\hline Cancer & $1(2.8 \%)$ & $1(7.7 \%)$ & $0(0.0 \%)$ & 0.361 \\
\hline Metastatic cancer & $0(0.0 \%)$ & $0(0.0 \%)$ & $0(0.0 \%)$ & - \\
\hline Leukaemia & $1(2.8 \%)$ & $1(7.7 \%)$ & $0(0.0 \%)$ & 0.361 \\
\hline Lymphoma & $1(2.8 \%)$ & $0(0.0 \%)$ & $1(4.3 \%)$ & 0.999 \\
\hline Clinical frailty score, median (IQR) & $3(2-3)$ & $3(3-3)$ & $2(2-3)$ & 0.057 \\
\hline \multicolumn{5}{|l|}{ Support before ECMO } \\
\hline Invasive ventilation & $36(100.0 \%)$ & $13(100.0 \%)$ & $23(100.0 \%)$ & - \\
\hline Prone positioning & $8(22.2 \%)$ & $1(7.7 \%)$ & $7(30.4 \%)$ & 0.213 \\
\hline Recruitment manoeuvre & $5(16.7 \%)$ & $1(11.1 \%)$ & $4(19.0 \%)$ & 0.999 \\
\hline Neuromuscular blocking agents & $17(48.6 \%)$ & $5(41.7 \%)$ & $12(52.2 \%)$ & 0.725 \\
\hline Norepinephrine & $27(77.1 \%)$ & $10(83.3 \%)$ & $17(73.9 \%)$ & 0.685 \\
\hline Renal replacement therapy & $6(17.1 \%)$ & $2(16.7 \%)$ & $4(17.4 \%)$ & 0.999 \\
\hline \multicolumn{5}{|l|}{ Vital signs before ECMO, median (IQR) } \\
\hline Temperature, ${ }^{\circ} \mathrm{C}$ & $36.9(36.4-37.6)$ & $36.8(36.3-37.4)$ & $37.0(36.4-37.6)$ & 0.495 \\
\hline Heart rate, beats/min & $96.0(87.0-110.5)$ & $95.5(89.5-106.5)$ & $100.0(85.0-116.5)$ & 0.626 \\
\hline Mean arterial pressure, $\mathrm{mmHg}$ & $76.0(69.0-82.5)$ & $81.0(77.5-85.8)$ & $73.0(68.0-81.5)$ & 0.081 \\
\hline $\mathrm{SpO}_{2}, \%$ & $91.5(87.2-95.8)$ & $91.0(87.5-95.2)$ & $92.0(87.2-95.8)$ & 0.610 \\
\hline \multicolumn{5}{|l|}{ Ventilatory variables before ECMO, median (IQR) } \\
\hline Tidal volume, mL/kg PBW & $5.3(4.0-6.5)$ & $5.1(4.6-5.9)$ & $5.6(3.8-6.5)$ & 0.958 \\
\hline Peak pressure, $\mathrm{cmH}_{2} \mathrm{O}$ & $29.0(24.0-35.0)$ & $27.5(23.5-31.0)$ & $31.0(25.0-36.0)$ & 0.209 \\
\hline PEEP, $\mathrm{cmH}_{2} \mathrm{O}$ & $12.0(10.0-15.0)$ & $12.0(10.0-15.0)$ & $12.0(10.0-15.0)$ & 0.880 \\
\hline Total respiratory rate, breaths/min & $22.0(16.5-26.0)$ & $26.0(23.0-30.0)$ & $20.0(12.0-23.0)$ & 0.004 \\
\hline Driving pressure, $\mathrm{CmH}_{2} \mathrm{O}$ & $16.0(10.0-24.0)$ & $15.0(11.0-19.5)$ & $19.0(10.0-24.0)$ & 0.274 \\
\hline $\mathrm{FiO}_{2}, \%$ & $100.0(80.0-100.0)$ & $100.0(80.0-100.0)$ & $100.0(85.0-100.0)$ & 0.970 \\
\hline
\end{tabular}




\begin{tabular}{|c|c|c|c|c|}
\hline & Overall & COVID-19 & Viral pneumonitis & $P$ \\
\hline \multicolumn{5}{|l|}{ Laboratory tests before ECMO, median (IQR) } \\
\hline $\mathrm{pH}$ & $7.24(7.13-7.34)$ & $7.28(7.20-7.34)$ & $7.20(7.12-7.34)$ & 0.358 \\
\hline $\mathrm{PaO}_{2}: \mathrm{FiO}_{2}$ & $83.0(67.0-115.0)$ & $87.9(67.0-108.3)$ & $83.0(67.0-118.0)$ & 0.808 \\
\hline $\mathrm{PaCO}_{2}, \mathrm{mmHg}$ & $67.0(52.0-76.0)$ & $70.5(55.7-76.0)$ & $66.0(52.0-75.0)$ & 0.640 \\
\hline Lactate, $\mathrm{mmol} / \mathrm{L}$ & $1.3(0.9-1.7)$ & $1.6(1.2-1.7)$ & $1.2(0.9-1.7)$ & 0.301 \\
\hline \multicolumn{5}{|l|}{ Hospital outcomes } \\
\hline \multicolumn{5}{|l|}{ Complications during ECMO } \\
\hline - Renal replacement therapy & $23(65.7 \%)$ & $8(61.5 \%)$ & $15(68.2 \%)$ & 0.726 \\
\hline - Pneumothorax & $3(8.3 \%)$ & $2(15.4 \%)$ & $1(4.3 \%)$ & 0.539 \\
\hline - Cardiac arrest & $3(8.3 \%)$ & $0(0.0 \%)$ & $3(13.0 \%)$ & 0.288 \\
\hline - Major bleeding & $8(22.2 \%)$ & $5(38.5 \%)$ & $3(13.0 \%)$ & 0.107 \\
\hline - Pulmonary embolism & $1(2.8 \%)$ & $1(7.7 \%)$ & $0(0.0 \%)$ & 0.361 \\
\hline - CNS diffuse ischaemia & $0(0.0 \%)$ & $0(0.0 \%)$ & $0(0.0 \%)$ & - \\
\hline - CNS bleeding & $2(5.6 \%)$ & $0(0.0 \%)$ & $2(8.7 \%)$ & 0.525 \\
\hline - Lung infection & $17(47.2 \%)$ & $5(38.5 \%)$ & $12(52.2 \%)$ & 0.502 \\
\hline - Thrombosis of ECMO circuit & $1(2.8 \%)$ & $0(0.0 \%)$ & $1(4.3 \%)$ & 0.999 \\
\hline Thrombosis of ECMO filter & $0(0.0 \%)$ & $0(0.0 \%)$ & $0(0.0 \%)$ & - \\
\hline Duration of ECMO, days, median (IQR) & $9.8(6.7-15.8)$ & $14.8(8.3-23.4)$ & $9.1(7.0-12.7)$ & 0.301 \\
\hline Duration of ventilation, days, median (IQR) & $18.8(13.3-46.0)$ & $31.2(17.1-54.0)$ & $16.7(10.1-31.0)$ & 0.043 \\
\hline ICU length of stay, days, median (IQR) & $30.4(19.5-50.1)$ & $48.0(33.0-106.1)$ & $23.8(15.8-35.0)$ & 0.004 \\
\hline In survivors, days, median (IQR) & $34.6(24.2-56.3)$ & $49.9(41.8-123.9)$ & $27.0(18.9-35.0)$ & 0.008 \\
\hline Hospital length of stay, days, median (IQR) & $40.5(23.7-59.2)$ & $57.2(48.9-143.9)$ & $30.7(19.0-46.1)$ & 0.005 \\
\hline In survivors, days, median (IQR) & $49.0(32.1-78.2)$ & $66.6(55.0-169.9)$ & $36.1(21.2-46.1)$ & 0.006 \\
\hline - ICU mortality & $11(31.4 \%)$ & $3(23.1 \%)$ & $8(36.4 \%)$ & 0.478 \\
\hline - Hospital mortality & $11(32.4 \%)$ & $3(23.1 \%)$ & $8(38.1 \%)$ & 0.465 \\
\hline - 180-day mortality & $11(33.3 \%)$ & $3(25.0 \%)$ & $8(38.1 \%)$ & 0.703 \\
\hline \multicolumn{5}{|l|}{ Long term outcomes at 6 months } \\
\hline WHODAS score, \%, median (IQR) & $22.9(4.2-50.0)$ & $32.3(8.3-56.2)$ & $22.9(3.1-42.7)$ & 0.392 \\
\hline - Disability & $8(47.1 \%)$ & $3(50.0 \%)$ & $5(45.5 \%)$ & 0.999 \\
\hline - New disability & $8(61.5 \%)$ & $4(66.7 \%)$ & $4(57.1 \%)$ & 0.999 \\
\hline EQ-5D-5L utility score, median (IQR) & $0.8(0.4-0.8)$ & $0.6(0.3-0.8)$ & $0.8(0.7-0.9)$ & 0.339 \\
\hline
\end{tabular}

APACHE = Acute Physiology and Chronic Health Evaluation; CNS = central nervous system; EQ-5D-5L = Five-level EuroQol five dimensions questionnaire; $\mathrm{FiO}_{2}=$ fraction of inspired oxygen; ICU = intensive care unit; IQR = interquartile range; $\mathrm{PaCO} \mathrm{C}_{2}=$ arterial partial pressure of carbon dioxide; $\mathrm{PaO}_{2}=$ arterial partial pressure of oxygen; PEEP = positive end-expiratory pressure; $\mathrm{PBW}^{2}=$ predicted body weight; SOFA = Sequential Organ Failure Assessment; $\mathrm{SpO}_{2}=$ oxygen saturation measured by pulse oximetry; $\mathrm{VA}-\mathrm{ECMO}=$ veno-arterial extracorporeal membrane oxygenation; $\mathrm{VV}-\mathrm{ECMO}=$ venovenous extracorporeal membrane oxygenation; WHODAS = World Health Organization Disability Assessment Schedule.

intubation. Traditionally, VV-ECMO outcomes have been worse for this late initiation group, ${ }^{2}$ and recent COVID-19 Extracorporeal Life Support Organization (ELSO) guidelines suggest late ( $>7$ days mechanical ventilation) initiation is a relative contraindication. ${ }^{3}$ However, we found that 6 -month mortality and disability were comparable for COVID-19 and viral pneumonitis patients, and compared favourably with other recently published data, ${ }^{4}$ suggesting that acceptable survival can occur in this late initiation group. In contrast, ECMO duration, ICU and hospital length of stay were longer with COVID-19, which may have significant resource implications during the COVID-19 pandemic and in periods of ICU strain. The findings of this study are preliminary, with small patient numbers and relatively few patients who were proned, and reflect a health care system that was not overwhelmed.

In conclusion, our data show that 6-month survival, disability, and quality of life for COVID-19 were similar to other viral pneumonitis, despite ECMO being initiated much later. However, the prolonged length of stay for these patients may have significant resource implications. These findings require further validation in larger datasets.

Acknowledgements: The EXEL Investigators are: Carol L Hodgson, 1,2,3,4 Alisa M Higgins, ${ }^{1,2}$ Michael J Bailey, ${ }^{1,2,4}$ Stephen 


\section{ORIGINAL ARTICLES}

Bernard, ${ }^{2}$ Bentley J Fulcher, ${ }^{1,2}$ Denise Koe, ${ }^{2}$ Natalie J Linke, ${ }^{1,2}$ Shannah Anderson, ${ }^{1}$ Stephen Bernard, ${ }^{3}$ Jasmin $V$ Board, ${ }^{3}$ Daniel Brodie, ${ }^{5}$ Heidi Buhr, ${ }^{6}$ Aidan JC Burrell, ${ }^{1,2,3}$ David J Cooper, ${ }^{1,2,3}$ Eddy Fan, ${ }^{7}$ John F Fraser, ${ }^{8,9}$ David J Gattas, ${ }^{6}$ Ingrid K Hopper, ${ }^{2}$ Sue Huckson, ${ }^{10}$ Edward Litton, ${ }^{11}$ Shay P McGuinness, ${ }^{1,12,13}$ Priya Nair, ${ }^{14}$ Neil Orford, ${ }^{15,16}$ Rachael L Parke, ${ }^{1,12,13,17}$ Vincent A Pellegrino, ${ }^{3}$ David V Pilcher, ${ }^{1,3}$ Jayne Sheldrake, ${ }^{3}$ Benjamin AJ Reddi, ${ }^{18}$ Dion Stub, ${ }^{2,3}$ Tony $\vee$ Trapani, ${ }^{1,2}$ Andrew A Udy, ${ }^{1,2,3}$ Ary Serpa-Neto ${ }^{1,4}$

1 Australian and New Zealand Intensive Care Research Centre, Monash University, Melbourne, VIC, Australia.

2 School of Public Health and Preventive Medicine, Monash University, Melbourne, VIC, Australia.

3 Intensive Care Unit, Alfred Hospital, Melbourne, VIC, Australia.

4 University of Melbourne, Melbourne, VIC, Australia.

5 Department of Medicine and Center for Acute Respiratory Failure, Columbia University College of Physicians and Surgeons/New York-Presbyterian Hospital, New York, NY, USA.

6 Intensive Care Unit, Royal Prince Alfred Hospital, Sydney, NSW, Australia.

7 Interdepartmental Division of Critical Care Medicine, University of Toronto, Toronto, Ontario, Canada.

8 University of Queensland, Brisbane, QLD, Australia.

9 Critical Care Research Group, Adult Intensive Care Society, Prince Charles Hospital, Brisbane, QLD, Australia.

10 Australian and New Zealand Intensive Care Society, Melbourne, VIC, Australia.

11 Intensive Care Unit, Fiona Stanley Hospital, Perth, WA, Australia.

12 Medical Research Institute of New Zealand, Wellington, New Zealand.

13 Cardiothoracic and Vascular Intensive Care Unit, Auckland City Hospital, Auckland, New Zealand.

14 Intensive Care Unit, St Vincent's Hospital, Sydney, NSW, Australia.

15 Intensive Care Unit, University Hospital Geelong, Geelong, VIC, Australia.

16 School of Medicine, Deakin University, Geelong, VIC, Australia

17 Faculty of Medical and Health Sciences, University of Auckland, Auckland, New Zealand.

18 Intensive Care Unit, Royal Adelaide Hospital, Adelaide, SA, Australia.

\section{Competing interests}

All authors declare that they do not have any potential conflict of interest in relation to this manuscript.

\section{Author details}

Aidan JC Burrell1,2

Ary Serpa Neto ${ }^{1}$

Andrew Udy ${ }^{1,2}$

Vincent Pellegrino 2

Carol Hodgson ${ }^{1,2}$

1 Australian and New Zealand Intensive Care Research Centre, School of Public Health and Preventive Medicine, Monash University, Melbourne, VIC, Australia.

2 Department of Intensive Care and Hyperbaric Medicine, Alfred Hospital, Melbourne, VIC, Australia.

Correspondence: aidan.burrell@monash.edu

doi: https://doi.org/10.51893/2022.1.OA10

\section{References}

1 Burrell AJ, Pellegrini B, Salimi F, et al. Outcomes for patients with COVID-19 admitted to Australian intensive care units during the first four months of the pandemic. Med J Australia 2021; 214: 23-30.

2 Schmidt M, Bailey M, Sheldrake J, et al. Predicting survival after extracorporeal membrane oxygenation for severe acute respiratory failure. The Respiratory Extracorporeal Membrane Oxygenation Survival Prediction (RESP) Score. Am J Respir Crit Care Med 2014; 189: 1374-82.

3 Badulak J, Antonini MV, Stead CM, et al. Extracorporeal membrane oxygenation for COVID-19: updated 2021 guidelines from the Extracorporeal Life Support Organization. ASAIO J 2021; 67: 485-95.

4 Barbaro RP, MacLaren G, Boonstra PS, et al. Extracorporeal membrane oxygenation for COVID-19: evolving outcomes from the international Extracorporeal Life Support Organization Registry. Lancet 2021; 398: 1230-8. 